\title{
Construire à plusieurs la machine à jouer
}

Figures de l'invention technique dans La Dispute mise en scène par

Patrice Chéreau

\section{Marie-Madeleine Mervant-Roux}

\section{(2) OpenEdition}

1 Journals

Édition électronique

URL : http://journals.openedition.org/genesis/1525

DOI : $10.4000 /$ genesis. 1525

ISSN : 2268-1590

Éditeur :

Presses universitaires de Paris Sorbonne (PUPS), Société internationale de génétique artistique littéraire et scientifique (SIGALES)

Édition imprimée

Date de publication : 27 novembre 2015

Pagination : 73-90

ISBN : 9791023105049

ISSN : 1167-5101

\section{Référence électronique}

Marie-Madeleine Mervant-Roux, « Construire à plusieurs la machine à jouer », Genesis [En ligne], 41 |

2015, mis en ligne le 09 mai 2017, consulté le 10 décembre 2020. URL : http://

journals.openedition.org/genesis/1525; DOl : https://doi.org/10.4000/genesis.1525 


\title{
Construire à plusieurs la machine à jouer Figures de l'invention technique dans La Dispute mise en scène par Patrice Chéreau
}

\author{
Marie-Madeleine Mervant-Roux
}

$\mathrm{L}$

e théâtre est une pratique collective. Exceptionnels sont les cas où les différentes fonctions exigées par cet art (définition d'un espace à part, élaboration d'un matériau textuel, élaboration d'un jeu scénique) sont assumées par une seule personne. Nombreux sont au contraire les cas où l'une ou l'autre de ces fonctions engage un grand nombre de contributeurs. Cependant, si la critique génétique, après avoir intégré à côté de l'auteur de l'œuvre dramatique la figure du metteur en scène ${ }^{1}$, a pris acte de l'hétérogénéité d'une production dont le résultat est une «conjonction fugace de texte, de posture et de souffle 2 » et reconnu que le scénographe, le dramaturge, les comédiens... pouvaient y contribuer, parfois de façon décisive ${ }^{3}$, le schéma d'une création due pour l'essentiel à deux grandes figures auctoriales n'en a pas été fondamentalement modifié. Quant à l'organisation matérielle de la représentation, souvent traduite par le terme générique de " régie ", les études théâtrales elles-mêmes ne lui ont guère porté attention en tant que telle : la conception (du décor, de la lumière, du son...) a été inscrite dans la sphère de la mise en scène, alors que la réalisation, c'est-à-dire la construction, l'installation, la mise en marche et la gestion des différents éléments scéniques n'a que très exceptionnellement fait l'objet de descriptions précises en dehors de publications techniques destinées aux praticiens. Pourtant, la dimension machinique, présente dans le théâtre grec, importante dans les jeux dramatiques médiévaux, reine des spectacles « spectaculaires » et triomphante dans l'opéra du XIX $x^{e}$ siècle, a connu au milieu du XXe siècle une revalorisation remarquable. Trois modèles politiques concurrents ont en ce domaine marqué la pensée théâtrale en France : le modèle brechtien, le modèle vilarien, le modèle de la « création collective ». Chacun à sa façon, ils ont remis la « régie » et les tâches traditionnellement considérées

1. Almuth Grésillon, «De l'écriture du texte de théâtre à la mise en scène », dans Cahiers de praxématique, $\mathrm{n}^{\circ} 26$, «Les mots et la scène », Praxiling, Montpellier III, Université Paul-Valéry, 1996, p. 71-93, repris sous une forme renouvelée dans La Mise en auvre. Itinéraires génétiques, Paris, CNRS Éditions, coll. « Textes et manuscrits », 2008, chap. XI, « Genèses théâtrales », p. 245-267.

2. Genesis, $n^{\circ}$ 26, « Théâtre », dir. Nathalie Léger et Almuth Grésillon, 2006.

3. Genèses théâtrales, dir. Almuth Grésillon, Marie-Madeleine Mervant-Roux, Dominique Budor, Paris, CNRS Éditions, coll. « Textes et manuscrits », 2010. 
comme subalternes au centre du processus théâtral. Mais sauf exceptions concernant surtout le troisième modèle (incarné en particulier par le Théâtre du Soleil) et alors que les « répétitions », avec les acteurs au travail, faisaient l'objet de plusieurs ouvrages ${ }^{4}$, les « acteurs » de l'ombre n'ont été étudiés que par des économistes ou spécialistes du management ${ }^{5}$. L'approche génétique, parce qu'elle a initialement abordé le théâtre par l'œuvre dramatique (sur le modèle de la littérature) et non par le dispositif spatial, n'a pas intégré la dimension technicienne et technologique de cet art comme elle a su le faire pour l'architecture et le cinéma.

Nous avons choisi de nous intéresser à ces participants ignorés, appréhendés de façon floue comme un collectif d'exécutants occupés à une activité foncièrement coopérative : les constructeurs, machinistes, électriciens, ouvriers des ateliers ou du plateau, techniciens et régisseurs. Faisant l'hypothèse, après Louis Jouvet et d'autres metteurs en scène ayant connu dans leurs débuts l'expérience de la régie, que ces pratiques non seulement peuvent être inventives, mais qu'elles peuvent porter le "sentiment du dramatique » aussi bien ou mieux que celles des acteurs 6 , nous avons tenté de reconstituer la vie de ce sous-ensemble mal connu à l'intérieur du grand ensemble « collaboratif » que suppose la genèse de tout spectacle théâtral. Nous avons concentré toute notre attention sur ce groupe. Nous ne parlerons pas de ceux qui contribuent au travail sur le texte, pas non plus des comédiens, ni des responsables des costumes, ni de toute la partie, essentielle elle aussi, de l'administration-gestion. Nous laisserons même de côté la conception technique lorsqu'elle est dissociée de la réalisation (un scénographe qui ne participe pas lui-même à la fabrication du décor). Nos observations concerneront les demi-visibles (les concepteursréalisateurs), les presque invisibles (les régisseurs), et les vrais invisibles (constructeurs, machinistes, électriciens, etc.). Nous n'avons pas pris pour exemple une production pour laquelle la dimension de création collective aurait été affichée, mais au contraire un spectacle qui passe pour être l'une des œuvres majeures d'un très grand metteur en scène. Une œuvre magistralement auctoriale, donc. Pourquoi ce choix ? Parce que plusieurs techniciens qui avaient travaillé pour ce spectacle - et d'autres du même metteur en scène - ont dit depuis longtemps avec des mots qui ne trompent pas qu'ils avaient eu le sentiment de contribuer personnellement à l'œuvre et même, chacun dans son domaine, de « réinventer le théâtre ${ }^{7}$ ». Nous avons récemment pu rencontrer plusieurs de ces praticiens.

\footnotetext{
4. Georges Banu (dir.), Les Répétitions. De Stanislavski à aujourd' hui, Arles, Actes Sud/Alternatives théâtrales/ Académie Expérimentale du théâtre, coll. «Le temps du théâtre », 2005.

5. Marc Jacquemond, Équipes de scène. L'organisation et le management des services techniques du spectacle vivant, Paris, Éditions AS, coll. « Scéno+ », 2009 ; Daniel Urrutiaguer, Économie et droit du spectacle vivant en France, Paris, Presses de la Sorbonne nouvelle, 2009 et Les Professions du spectacle vivant. Entre les logiques du marché et du service public, Paris, Armand Colin, 2012. Voir aussi ce dossier récent : Théâtre/Public, $\mathrm{n}^{\circ} 217$, « Théâtre en travail. Mutations des métiers du spectacle (toujours) vivant », dir. Martial Poirson et Emmanuel Wallon, juin 2015. 6. «Quand je considère les gens de théâtre, c'est le machiniste qui m'apparaît, pour avoir, mieux qu'un autre, le sens du dramatique [...]. L'acteur, s'il joue, n'a de sentiment que pour lui-même [...]. Il n'en est pas de même de qui travaille pour le décor [...] », Louis Jouvet, « Découverte de Sabbatini », préface à Nicola Sabbattini, Pratiques pour fabriquer scènes et machines de théâtre, Zurich, Ides et Calendes, 1942 [rééd. 1978], p. XXXVI-XXXVII.

7. Voir par exemple Yves Bernard, «Tout commence par un récit », Des coulisses et des cintres, Saint-Brieuc, Théâtre s en Bretagne, $n^{\circ}$ 9, $1^{\text {er }}$ tri. 2001, p. 4-8; André Serré, « De l'image au paysage sonore, la passion du théâtre public », Agôn, Enquêtes, Souvenirs de théâtre, TNP ; URL : <http://agon.ens-lyon.fr/index.php?id=2615>.
} 
Eux-mêmes n'avaient pas conservé de traces matérielles de leur travail. Nous leur avons soumis quelques documents empruntés aux archives du metteur en scène, documents qui les concernaient, mais qu'ils n'avaient jamais vus, afin d'interroger leur mémoire. Leurs témoignages, ajoutés à d'autres, plus anciens, nous ont confortée dans notre hypothèse selon laquelle la fonction de «l'équipe technique » (selon la formule courante) n'est pas de simple exécution et nous ont invitée à prendre de la distance par rapport à la deuxième idée fréquemment entendue à son sujet : le caractère - ontologiquement ? - « collectif » de son fonctionnement.

\section{Présentation du spectacle}

Patrice Chéreau a monté La Dispute, un texte bref, tardif et rarement joué de Marivaux, en 1973. La création a eu lieu le 24 octobre à Paris au Théâtre de la Musique (ancienne Gaîté lyrique).

En plein cœur du XviII e siècle, un couple de souverains éclairés, princes d'un État idéal, tente, pour clore une dispute anodine, de remonter aux sources du comportement amoureux. Ils se donnent alors le spectacle d'une expérience menée avec quatre sujets des deux sexes, séquestrés à cette seule fin depuis leur plus jeune âge et élevés dans l'isolement le plus complet par des serviteurs noirs 8 .

Dans le spectacle, la pièce est précédée de deux longues séquences : l'écoute intégrale (7'), rideau fermé, de l'Ode funèbre maçonnique de Mozart (dans la version de Bruno Walter), alors que des fumées montent de la fosse d'orchestre vide, puis un prologue, qui fait dialoguer le prince, la princesse et ses suivantes et se joue dans la salle, sur un praticable 9 de quatre mètres de côté élevé au milieu des fauteuils. Les répliques sont empruntées à d'autres textes de Marivaux, choisies et montées par François Regnault. La Cour traverse ensuite la fosse sur une planche jetée par le prince (par l'acteur Roland Bertin, aidé discrètement par un machiniste), pénètre sur le plateau, ouvre le rideau, laissant apparaître une sorte de clairière sombre (une épaisse forêt occupe le fond de scène), avec de très hauts murs percés de portes et de fenêtres qui semblent les restes d'un palais abandonné. Le sol est terreux, on aperçoit une mare pleine d'eau. En sept nuits - un découpage conçu par Chéreau -, les deux filles (Laurence Bourdil, Hermine Karagheuz) et les deux garçons (Alain Libolt, Hugues Quester) vivent le désir, le conflit, la trahison et la blessure, observés par les serviteurs (les chanteurs Mabel King et Thomas Anderson) et par la Cour. Le décor, dû à Richard Peduzzi, est animé de l'intérieur par les éclairages d'André Diot et par les sons d'André Serré. Durant certaines séquences, les «maisons » se déplacent, comme si elles étaient animées d'une vie étrange et inquiétante (fig. 1).

Cette création se situe à un moment clé du parcours de Chéreau. Après quelques contributions (comme machiniste, acteur et décorateur) aux activités de la troupe du lycée Louis-le-Grand, ses premières mises en scène dans le cadre du théâtre universitaire (1964-1966), la direction du Théâtre de Sartrouville (1966-1969) conclue par une faillite et l'exil en Italie (1969-1972), marqué par plusieurs créations, entre Spolète (festival des

8. Résumé de la pièce rédigé par $\mathrm{P}$. Chéreau pour le programme du spectacle.

9. Plate-forme pouvant supporter le poids et le mouvement des comédiens (voir fig. 5).

\section{La Dispute et ses techniciens}




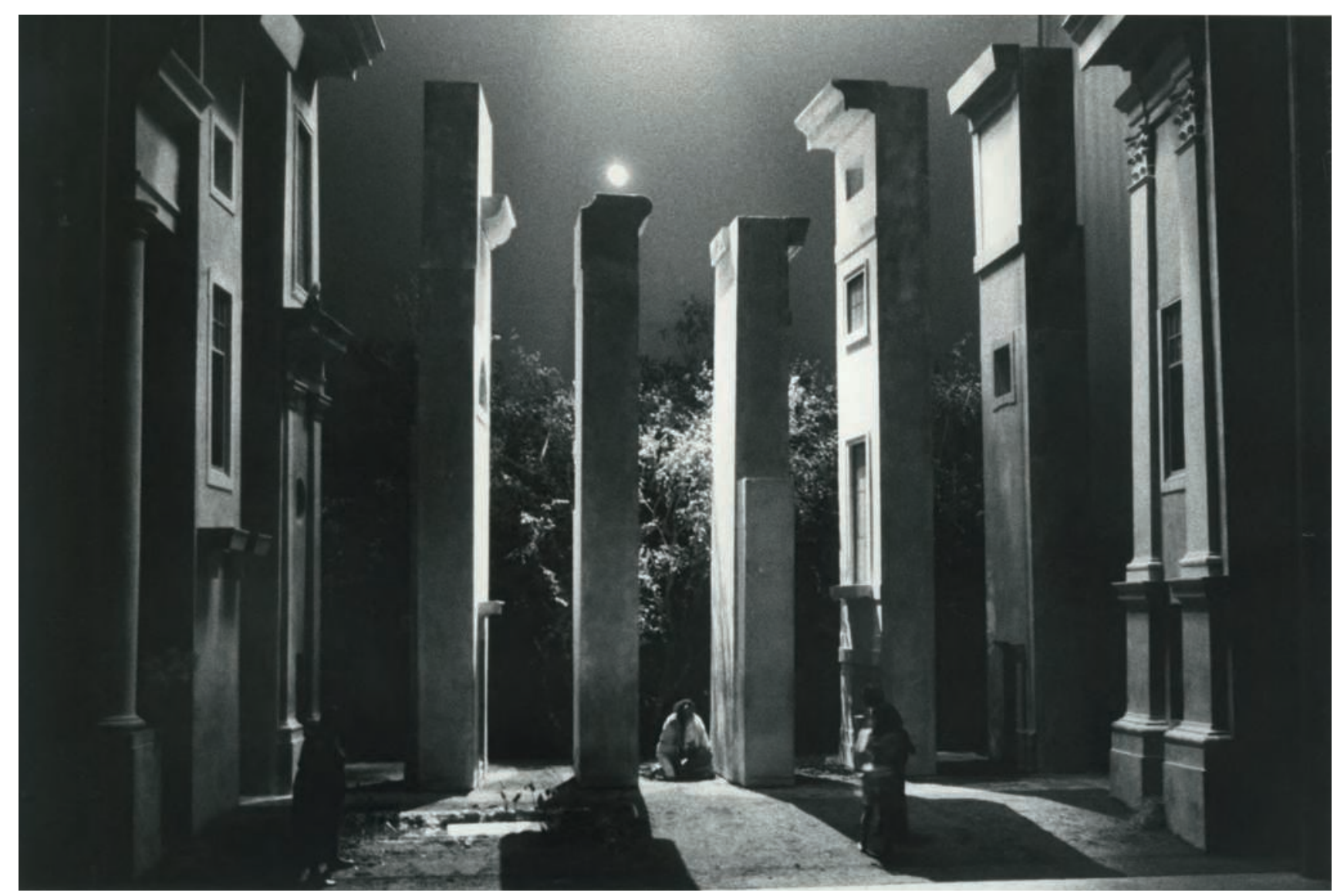

Fig. 1 : La Dispute. Les « maisons » et la forêt (décor de Richard Peduzzi, éclairages d'André Diot), Paris, Théâtre de la Musique, 1973

Photographie de Claude Bricage (C) Claude Bricage

Deux Mondes) et Milan (Piccolo Teatro), il vient d'être nommé (en mars 1972) codirecteur du tout nouveau TNP de Villeurbanne où il crée Massacre à Paris en mai 1972, puis Toller en janvier 1973. Il a 29 ans ${ }^{10}$.

Le terme de « machine à jouer », utilisé par Patrice Chéreau à propos des Soldats, en 1966, et de Don Juan, en 1969, période où il conçoit lui-même ses décors, disparaît ensuite de son vocabulaire. Marqué par l'esthétique brechtienne, le concept était dû à René Allio, qui se référait à la «machine à habiter » de Le Corbusier. Si nous pouvons y avoir recours à propos de La Dispute, c'est que le René Allio de 1977, admirateur de ce spectacle, nous y autorise : «Même un décor fortement métaphorisé, explique-t-il, peut être considéré, dans un sens large, comme une machine à jouer11. »

Notre étude nous conduira à évoquer la première tournée du spectacle, dans sept villes de France, une gageure pour les techniciens, et la création en avril 1976, à Villeurbanne, d'une seconde version du spectacle, qui voyagera ensuite en France et en Europe.

10. Pour plus de détails, voir Odette Aslan (dir.), Chéreau, VCT, vol. XIV, Paris, Éditions du CNRS, coll. « Arts du spectacle », 1986; Michel Bataillon, Un défi en province. Chronique d'une aventure théâtrale : Chéreau, 1972-1982, Paris, Marval, 2005.

11. René Allio, entretien avec J.-P. Sarrazac, « De la machine à jouer au paysage mental », dans Travail théâtral, n $28-29,1977$, p. 107-112. Reproduit dans Double Jeu, n 4, « René Allio », 2004, p. 21-28. 


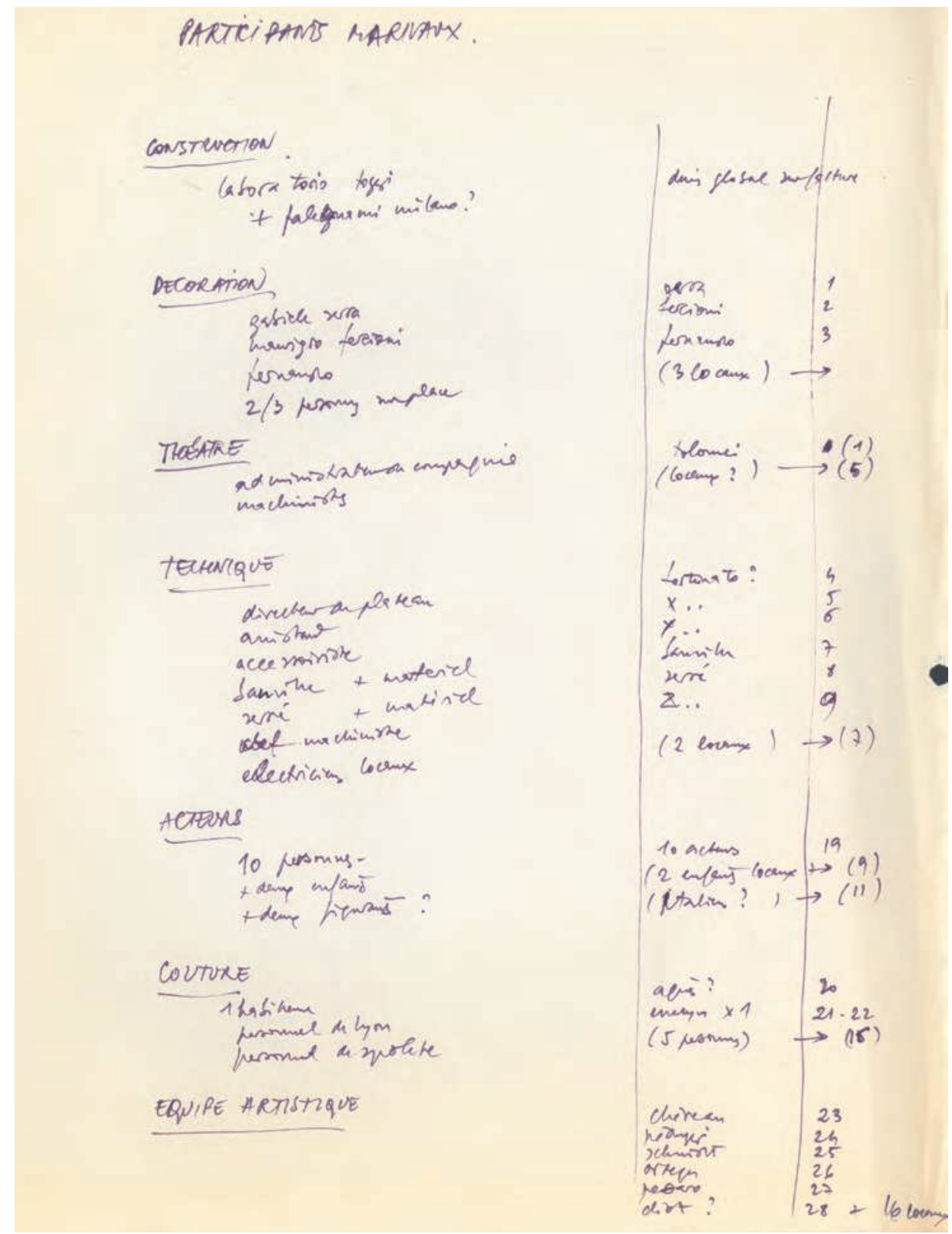

Fig. 2 : Tableau de la main de Patrice Chéreau intitulé «Participants Marivaux », non daté (probablement juin-juillet 1973) Fonds Patrice Chéreau/IMEC. La Dispute

\section{Une seconde « distribution»?}

Nous allons faire connaissance avec les techniciens de La Dispute à travers un document qui ne comporte pas tous leurs noms, loin s'en faut, mais qui éclaire utilement la genèse de ce qui ne sera jamais un collectif, ni même une équipe, plutôt une réunion de figures appartenant à trois univers : Sartrouville, l'épisode italien et Villeurbanne. Il s'agit d'un tableau en trois colonnes (fig. 2) intitulé « Participants Marivaux », écrit de la main de Chéreau, non daté, mais dont on peut, par recoupement, situer la rédaction à juin-juillet 1973 : Chéreau a entrepris de travailler sur le texte de La Dispute, il connaît bien les personnels italiens, mais aussi ceux du TNP (on peut lire les noms de Banville, chef électricien, et de Serré, régisseur son). 
Le tableau comporte verticalement sept rubriques. De haut en bas : "Construction », « Décoration », «Théâtre », « Technique », «Acteurs », « Couture » et «Équipe artistique ». Notons que les « artistes» (le metteur en scène et ses deux assistants, les concepteurs du décor, des costumes et de la lumière) sont les derniers nommés. Question de courtoisie. Pour le reste, Chéreau suit la chronologie présumée des tâches à accomplir à partir du début du travail théâtral lui-même : François Regnault n'apparaît pas dans ce décompte des "participants ", on entre bien dans l'« organisation matérielle de la représentation ». Les trois colonnes (avec des exceptions, nous y reviendrons) correspondent, de gauche à droite : à l'énoncé des fonctions ; aux noms des personnes envisagées ; au nombre d'embauches à effectuer, les "locaux », c'est-à-dire au départ les membres de l'équipe du TNP où se dérouleront les répétitions (« Lyon » vaut pour Villeurbanne), apparaissant entre parenthèses. La liste très ordonnée par laquelle Chéreau fixe ce qui semble être un premier état de sa réflexion personnelle sur sa "distribution » (au sens large) n'est cependant pas homogène, et cette hétérogénéité nous apprend quelque chose pour notre sujet.

Concernant la réalisation des décors (les deux premières rubriques) et l'organisation du plateau (les deux suivantes, qui couvrent les mouvements de la machinerie, l'éclairage et le son), le metteur en scène note immédiatement dans la colonne de gauche des noms d'ateliers précis, quelquefois des noms de personnes. Il se contente ensuite de reporter ces noms dans la deuxième colonne. Comme si la fonction technique officielle pesait moins que telle façon de faire particulière ou telle personnalité remarquable à travers lesquelles il pense déjà son spectacle. À la rubrique «Construction », il indique un atelier italien de Spolète ( « laboratorio Tozzi »), auquel s'ajoute, avec un point d'interrogation, « Falegnami Milano ». C'est bien en Italie que sera construit le décor, mais à Florence et par une autre équipe, dirigée par le chef machiniste du Théâtre La Pergola, Angelo Jotti, sollicité par Peduzzi. Le praticable en fer avec des rails de grand 8 pour les déplacements des maisons sera, lui, construit à Milan. Pour la « décoration » (la peinture), que Chéreau pense confier à des Italiens, avec un apport de trois personnes « sur place », elle sera finalement effectuée au TNP sous la direction de Vital Desbrousses et Marius Faucher. Danka Semenowicz, responsable des accessoires, des miroirs, des fumées, en sera responsable pour la seconde version, le travail étant alors effectué en Italie. Dans la rubrique «Technique », plusieurs indications doivent être commentées. En face de la fonction «directeur de plateau » est inscrit un simple prénom : " Fortunato ». Il s'agit du chef de plateau du Piccolo Teatro de Milan. Celui qui occupera finalement ce poste, Yves Bernard, collaborateur régulier de Chéreau depuis 1967 en tant que régisseur général, est alors en tournée à l'étranger. Il n'entrera qu'en septembre 1973 dans le projet (le décor est déjà construit) et assumera aussi dans La Dispute les fonctions de directeur technique et de chef machiniste - un découpage terminologique qu'il n'apprécie guère. À Spolète, il s'était épris de la culture technique des Italiens, ce qui lui permettra d'apporter dans le projet ce que symbolise dans la liste de 1973 la figure de "Fortunato ». Lorsqu'en 1976, par exemple, il faudra entièrement refaire le décor usé, il contactera « Tozzi », qui avait travaillé pour Visconti et dirigera une équipe mixte, incluant Rodolfo Grifoni, grand constructeur. Le serrurier du TNP, Bernard Steffenino, ira faire les serrureries, et le tour sera joué, moyennant quelques péripéties romanesques que nous raconterons plus loin. Banville, chef électricien du TNP, dont le nom 
est cité à la première colonne, s'avérera en effet important, entre autres parce que c'est lui qui embauchera les deux électriciens « locaux », dont Gérard Boucher, lequel participera aux dernières représentations parisiennes de 1973 comme poursuiteur ${ }^{12}$ et assurera la régie lumière à partir de 1976. Chéreau se soucie de toutes ces embauches apparemment modestes : plusieurs feuilles de notes (d'août-septembre 1973) témoignent de la recherche d'électriciens entre le Piccolo et Villeurbanne.

Quant à Serré (André), son inscription dans le tableau comme technicien son traduit le fait que Chéreau l'a sauvé de la mise à pied (régisseur général au TNP après avoir assumé une série d'autres fonctions, il était en délicatesse avec un supérieur). Celui qui élaborait à cette période le son de Toller, après celui de Massacre à Paris, développera pour La Dispute une collaboration si fructueuse avec le metteur en scène que son nom rejoindra dans le programme ceux des membres de l'équipe artistique.

Revenons pour finir à la troisième rubrique, "Théâtre », où l'on voit que les cinq machinistes prévus devaient être des «locaux ». En août-septembre 1973, Chéreau cite dans ses notes plusieurs noms de personnes possibles, y compris des Italiens, qui ne disparaissent donc pas du projet. Nous avons évoqué Rodolfo Grifoni. Parce qu'il travaille excellemment le cintre13 à l'italienne, il sera ramené de Spolète par Yves Bernard en 1976 pour la deuxième tournée, où cette technique légère sera précieuse. Nous reviendrons à travers l'exemple de Bernard Steffenino, le serrurier du TNP, sur ces constructeurs qui sont aussi des machinistes.

Quelles leçons tirer de ce document?

- Dès le début, Patrice Chéreau réfléchit en même temps à la dramaturgie, aux acteurs et à la régie, comme l'indique aussi le schéma des nuits (fig. 3). C'est que l'espace joue dans son théâtre un véritable rôle : le dispositif agit sur le spectateur (la fosse d'orchestre est construite là où elle n'existait pas) et la machinerie joue elle-même - « la maison le palais carnivore », note-t-il en août ou septembre 1973.

- Le metteur en scène s'intéresse aux compétences personnelles repérées ou espérées, et ceci à tous les niveaux. Richard Peduzzi et Yves Bernard, qui - rappelons-le - collaborent l'un et l'autre avec lui depuis 1967, incarnent dans le champ technique deux autres pôles d'autorité et d'expertise, des relais de confiance, qui influeront sur les embauches.

- Le tableau traduit une distinction nette et même une séparation stricte des tâches.

En résumé, la place majeure accordée par Chéreau à la « machine » scénique, qui s'incarne pour lui en un certain nombre de techniciens spécialisés et individualisés (dix-neuf, sans compter les constructeurs), constitue par certains côtés un élément favorable à l'épanouissement des habiletés techniques. L'éclatement des pratiques cependant, dont le metteur en scène tient seul les fils, semble contraire à l'émergence d'une contribution créatrice de la régie elle-même. Chéreau, selon André Serré, n’en aurait jamais accepté l'idée. C'est pourtant cette contribution spécifique que nous allons maintenant décrire à travers quelques exemples.

12. Ici, celui qui manipule la poursuite (projecteur mobile) pour suivre et éclairer les acteurs.

13. Partie du théâtre ou du dessus de la scène permettant d'escamoter les décors. 


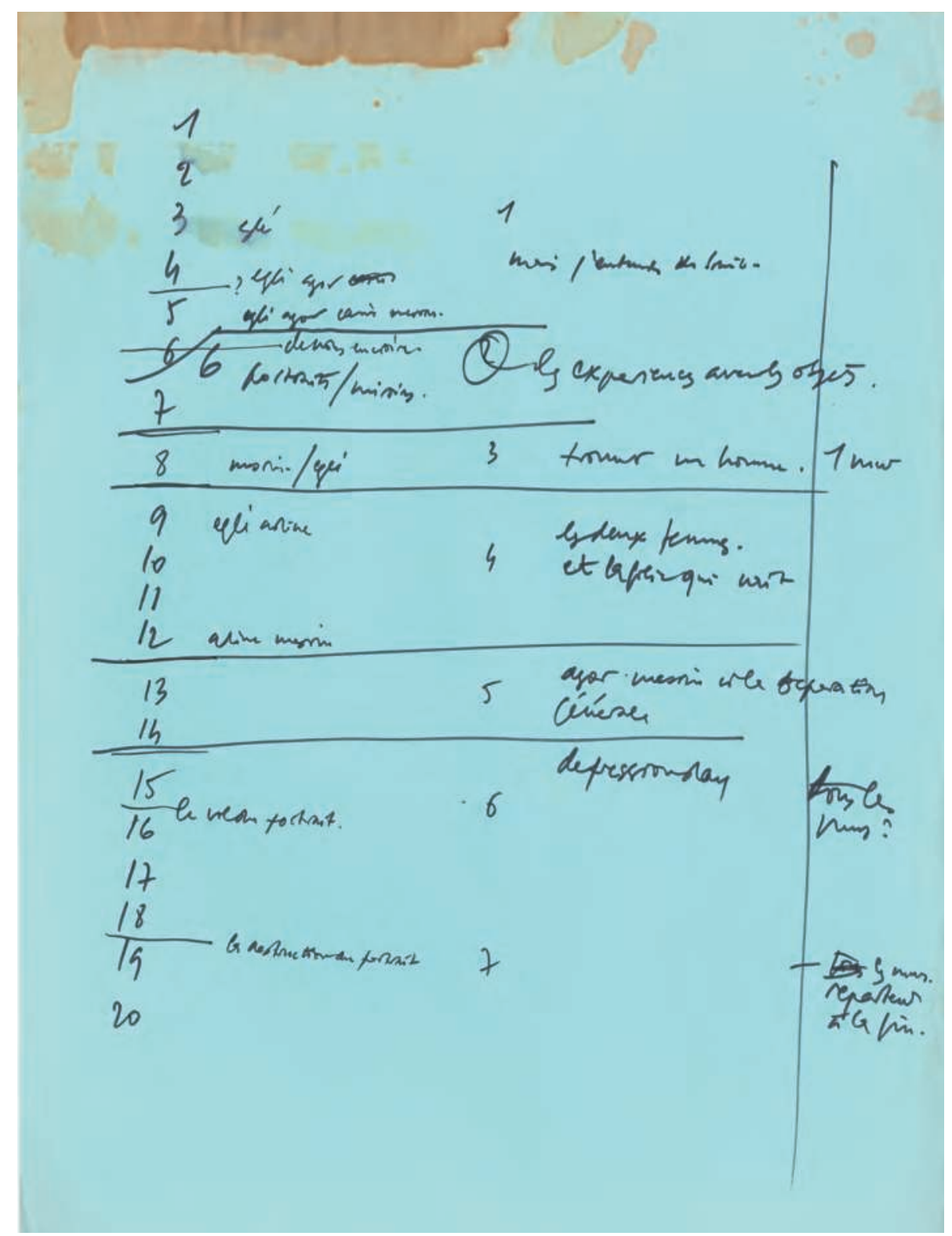

Fig. 3 : Schéma des sept nuits, de la main de Patrice Chéreau (sans titre, non daté [1973]. Â droite, les indications concernant les murs.

Fonds Patrice Chéreau/IMEC. La Dispute

\section{Imagination productrice technique et imaginaire théâtral}

\section{Innovations et inventions}

Les gestes et les usages d'appareils pionniers semblent avoir accompagné toute la genèse de La Dispute. Il fallait un camion, raconte Bernard Steffenino, pour transporter les matériels lumière et son, si originaux à l'époque qu'aucune salle n'en disposait. Nous évoquerons trois expérimentations technologiques, dont les deux premières sont aujourd'hui célèbres :

a) l'entrée et l'adaptation des fluos au théâtre. On a beaucoup dit qu'André Diot avait introduit les HMI 14 sur la scène, mais c'est au Piccolo Teatro que Chéreau avait vu ces lampes et c'est Yves Bernard qui en a trouvé chez Cremer (pour Lear, 1975). Ce que Diot a apporté de neuf, à l'occasion de La Dispute, c'est le tube fluo ${ }^{15}$, dont on pouvait

14. Les projecteurs HMI (halogène métallique iodure) sont constitués par des lampes à arc et produisent une lumière très blanche, supérieure à celle des lampes à incandescence des projecteurs plus traditionnels.

15. Les fluos (florescents), proches des néons, produisent une lumière blafarde. 
diminuer l'intensité sans modifier la couleur. Le problème majeur résidait dans l'absence de gradateur permettant une diminution régulière, ce qui a entraîné le développement de nouveaux gestes artisanaux et la création d'une petite régie particulière (voir ci-dessous, «Évolutions de la régie »,p. 84);

b) les premiers " silences habités ", faits de sons originaux mixés en direct au magnétophone (fig. 4 et 4 bis et leur commentaire par André Serré, p. 82);

c) la première "vraie forêt » scénique. Les troncs des arbres étaient vrais, les branches aussi (du moins au début). Yves Bernard découvrit chez un spécialiste de décoration florale un moyen de garder fraîches les feuilles : vider la sève et tremper les branches dans un bain de paraffine. Or ce traitement représentait un réel danger. "Nous avions fait des tests : quand nous mettions le feu aux feuilles, non seulement elles brûlaient, mais elles explosaient ! » L'effet de ces grands arbres était si réussi que les techniciens ont couru le risque, et découvert des gains inattendus de leur trouvaille. Ils évoquent encore aujourd'hui avec une vive émotion le début du spectacle et « le grand courant d'air qui traversait la salle à l'ouverture du velours rouge. On sentait une odeur de sous-bois étonnante 16 ».

À ces innovations, il faut ajouter les importations pertinentes, comme celle de la méthode italienne dans la construction des châssis : pour solidifier les murs verticaux, au lieu des châssis à plat, mortaisés, très fragiles, utilisés en France, Yves Bernard introduit le système de battants à champ, plus solide. Sans oublier les idées rocambolesques permettant de résoudre des difficultés a priori insurmontables. En 1976, par exemple, lorsque le décor s'avère inutilisable, le plus simple est de le faire refaire en Italie, mais les droits de douane sont exorbitants. Le directeur technique trouve la solution : les anciens éléments y seront transportés " pour réparation », et ce sont d'autres éléments, totalement neufs, qui reviendront à Villeurbanne.

Les difficultés successives, racontent les techniciens, représentaient autant de « défis » pour ceux qui avaient à les résoudre et entraient alors dans ce que Nicolas Dodier appelle le «théâtre » ou « les arènes » des « habiletés techniques 17 ». Le coup d'œil de Chéreau était décisif, mais l'audience de la prouesse était bien plus large. « Il y a des moments, dit Yves Bernard, des passages - de cinq minutes ou de deux semaines - où l'on est soudain la personne la plus importante du projet. C'est l'addition de ces moments qui fait que le spectacle existe $^{18}$. » On peut parler aussi pour certains techniciens d'un esprit ludique, traduit dans des blagues internes au groupe, comme l'installation dans la fosse d'orchestre d'un pupitre « magique » où les pages de la partition tournaient toutes seules, visibles d'une partie du public seulement ${ }^{19} \ldots$ Ces deux modes d'engagement, le grave et le léger, semblent avoir contribué à dynamiser la préparation de La Dispute et toutes les représentations. Leur développement autocontrôlé indique l'existence en chaque collaborateur d'un imaginaire du spectacle - on jouait à construire la machine à jouer -, ce qui sera éclairé par la rubrique suivante.

16. B. Steffenino à M.-M. Mervant-Roux (le 23 décembre 2014). Lui-même était à ce moment-là dans la fosse, où il récupérait les éléments du praticable démonté.

17. Nicolas Dodier, «Les arènes des habiletés techniques », dans Raisons pratiques, $\mathrm{n}^{\circ} 4$, « Les objets dans l'action », EHESS, 1993, p. 115-139.

18. Y. Bernard, loc. cit., p. 8.

19. Il faut ici noter le rôle de Danka Semenovicz, responsable des accessoires. 


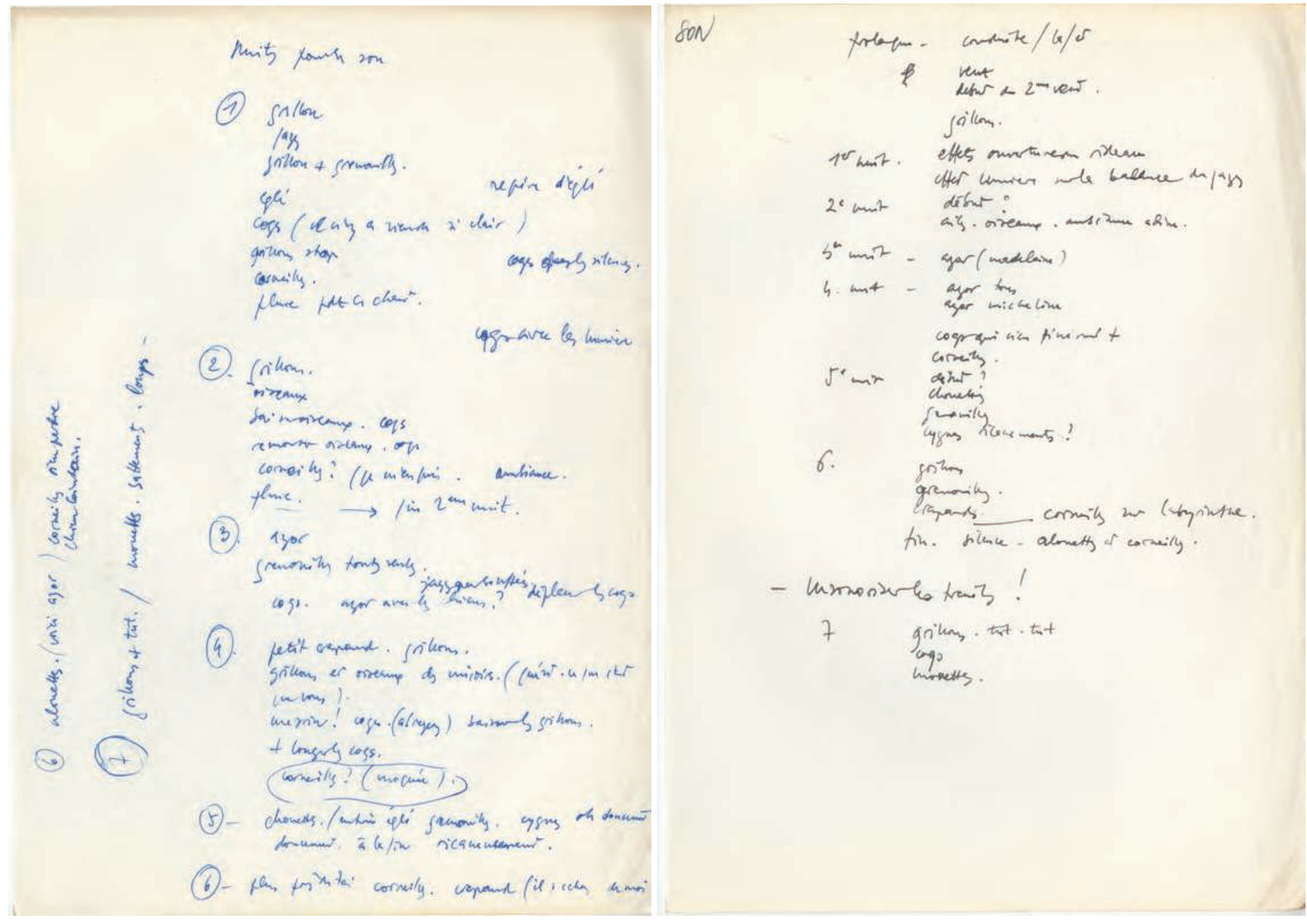

Fig. 4 et 4 bis : « Nuits pour le son », et «Son », notes non datées, de la main de P. Chéreau Fonds Patrice Chéreau/IMEC. La Dispute

\section{« Notes sur le son », par André Serré (décembre 2014) Commentaire des documents 4 et 4 bis}

Ces deux documents permettent d'expliquer la méthode de travail mise en place au début de ma collaboration avec Patrice Chéreau et de présenter le matériel employé à cette époque. Si par la suite Chéreau a maîtrisé complètement toutes les techniques, du fait de sa boulimie à tout savoir et sans doute aussi son désir de tout contrôler, pour Toller et La Dispute (1973), nous avons inventé ensemble une nouvelle manière d'intégrer le son au théâtre. Il s'agissait de produire des « faux silences » que par la suite j'ai nommés « les silences habités ». Patrice était parti d'une idée très simple : «Au cinéma, s’il y a un paysage sans personnage, on n'a pas le silence, on a les "sons seuls" du paysage. Pourquoi au théâtre, dans une image sans personnages, n'inventerions-nous pas des sons qui ne seraient pas le son de la scène vide? » Ainsi pour Toller, j'ai dû faire des boucles et des boucles de pluie fine que, quelquefois, seuls lui et moi entendions, mais qui faisaient « tourner » l'ambiance de la scène. Et puis des chiens au lointain, des horloges. Techniquement c'était difficile, l'informatique n'était pas encore au rendez-vous, il fallait enregistrer une longueur de pluie sur de la bande magnétique 6,35, que je scotchais pour en faire une boucle longue et que l'on faisait tourner à petite vitesse en calant la bande sur des pieds de micro dans un couloir. Car Patrice ne voulait pas entendre le raccord et à l'époque cela tenait du prodige. Il n'aimait pas les bruits de sonothèque, enregistrés à cette époque sur des disques vinyles. Pour La Dispute, il y avait sur le plateau une vraie forêt. Je lui ai proposé d'aller enregistrer les bruits dans 
la campagne, où j'habite encore. Je me suis procuré un Nagra $4 \mathrm{~S}$ et, armé de perches et de micros, je suis d'abord allé près de chez moi - c'était l'été - enregistrer les grillons. Je me souviens que la première fois où je les ai fait écouter à Patrice, il était fou de joie, il riait, comme souvent, comme s'il venait de faire une bonne blague : le son entrait au théâtre. Et il avait mis le doigt dans l'engrenage. Son avidité à vouloir toujours plus n'avait d'égale que son avidité à manger, y compris dans l'assiette de son voisin.

Armé de ce nouveau matériel, nous sommes partis de nuit dans le «parc des oiseaux » de Villars-les-Dombes, qui venait d'ouvrir. Ce parc se situe au milieu des étangs de la Dombes, mais en bordure d'une route très fréquentée à l'époque, parce qu'elle était la route de Genève pour les poids-lourds. Nous avons demandé l'autorisation d'entrer dans le parc la nuit. J'ai trouvé là la base de tous les bruitages de La Dispute. J'enregistrais des oiseaux que je ne voyais pas : ainsi les conversations des cygnes, dont le bruit de tuyau est très caractéristique et que Patrice utilisait comme des ricanements.

Un processus de travail s'est mis en place. En fin de matinée, chaque jour, Patrice montait dans la cabine son, je lui faisais écouter ce que j'avais enregistré la veille, il prenait des notes. Quelquefois, des sons entendus lui donnaient l'idée d'autres sons, qu'il me demandait pour le lendemain. Nous procédions de la même manière pour la musique : il arrivait avec des vinyles sous le bras, il me demandait de lui faire entendre un ou deux morceaux qu'il avait écoutés la veille et d'en extraire des petits morceaux pour la répétition de l'après-midi. Quelques sons retenus devaient être isolés très vite, parce que dans la même répétition, il avait envie de les proposer aux acteurs.

Les deux documents retracent exactement ce travail de collaboration, qui fonctionnait sur un échange et des allers-retours. J'imagine que le premier est une liste des sons entendus, qu'il essaie d'intégrer à différentes scènes en relation avec sa dramaturgie ; l'autre est une suite de remarques sur la conduite, à me transmettre. Au fil des répétitions, il a décidé que le son allait accentuer les effets lumière pour « construire » réellement les sept nuits.

Patrice ne s'intéressait pas vraiment à la manière dont serait diffusée la bande sonore. Tout cela, qui était très novateur, je l'ai proposé moi-même.

J'avais imaginé trois systèmes de diffusion : quatre enceintes de très grande qualité étaient placées dans la fosse d'orchestre. Ces enceintes Elipson, achetées pour l'occasion, diffusaient uniquement l'Ode funèbre maçonnique de Mozart qui servait d'Ouverture. Au lointain, à jardin et à cour, de chaque côté de la forêt, deux énormes enceintes servaient à diffuser le jazz qui accompagnait souvent l'arrivée des serviteurs noirs : des enceintes à deux voies Altec avec une trompette d'aigus. Enfin j'avais ceinturé le plateau de vingt-quatre petites enceintes Elipson, fabriquées spécialement pour moi par M. Léon, qui étaient des dérivées d'une enceinte commercialisée : la S10. Comme elles intégraient un HP de $8 \mathrm{~cm}$, elles ont été baptisées les S8. Ces enceintes étaient fixées au châssis du plateau et alimentées en quatre pistes, c'est-à-dire qu'une enceinte sur quatre donnait un son différent.

Je voulais, pour donner plus de relief à tous les petits sons enregistrés, que le mixage ne soit pas fait sur la bande sonore mais dans le volume du plateau. Ce qui permettait à Patrice d'intervenir. Si par exemple, dans une ambiance de nuit, il souhaitait que les fameuses « grenouilles tut-tut » soient moins présentes par rapport au bruit continu des grillons, je pouvais le faire en direct. Pour pouvoir diffuser tous ces sons, j'utilisais trois magnétophones Revox A77, ce qui me permettait d'avoir trois pistes stéréo disponibles. Un des magnétophones était réservé à la musique, les deux autres aux bruitages, ce qui laissait quatre pistes pour ces derniers. Cette technique devait s'affiner dans les spectacles suivants.

Quand Patrice Chéreau est arrivé à Villeurbanne, il n'utilisait pas la bande-son et avait même pour elle une certaine aversion. Dans Massacre à Paris (1972), les musiques étaient en direct, avec un petit orchestre qu'il faisait jouer soit en coulisses, soit dans les couloirs d'accès à la scène, afin de donner un effet d'éloignement à cour ou à jardin. Dans les trois spectacles suivants : Toller, La Dispute et Peer Gynt, nous avons affirmé notre technique d'accompagnement sonore, que nous avons repris dans tous les spectacles suivants et qui fera école, puisque la plupart des spectacles actuels continuent à utiliser ce système. 


\section{Une cocréation rythmique}

Les machinistes de La Dispute nous sont apparus comme des acteurs du mouvement scénique, au même titre que les comédiens, participant directement au réglage du rythme du spectacle, un élément clé de l'art de Chéreau. André Serré se rappelle la façon dont celuici vantait la prestesse et la réactivité des acteurs italiens et pense qu'il éprouvait la même admiration pour les machinistes italiens. Ce qui créait pour les Français une compétition implicite. "Plus lentement », "plus vite », énormément de notes du metteur en scène concernaient l'exactitude rythmique : celle des effets de lumière (les conduites des poursuiteurs étaient diaboliquement précises), celle des envois de cris, de bruits (effectués par André Serré). Nous avons choisi d'évoquer une troisième catégorie de gestes : ceux qu'exigeaient les « murs qui marchent 20 », engageant quatre machinistes, deux côté cour, deux côté jardin. De véritables répétitions avaient été nécessaires, d'abord avec les machinistes du TNP, puis avec ceux du Théâtre de la Musique - plus exactement, ceux du Théâtre de Chaillot, alors fermé pour travaux. "On faisait avancer les maisons à la main avec des treuils, raconte Bernard Steffenino (présent dans la version 1976), Julien Bordier, Fernand Ferrero, François Perez et moi, et il fallait aller assez vite. On était épuisés. Yves Bernard réglait l'ensemble : nous avions des boîtiers électriques avec des top rouges et des top verts. On ne bougeait pas les maisons n'importe comment, mais en relation avec la lumière, avec le jeu des ombres, ce n'était pas gratuit. Rien n'était gratuit ${ }^{21}$. » L'impression de mécanique devait être parfaite, les arrêts sans bavure. Lors de la première, le metteur en scène note : « fins nettes » [il veut des]. Mais le geste était travaillé comme un geste d'acteur : "Chéreau voulait que les gens le sentent, le respirent. » En cas de panne ou de maladresse, les machinistes étaient protégés. «Un jour un câble a cassé dans une maison, on a réparé à quatre pattes, pendant le spectacle. On était sous le plateau, le praticable avec les rails faisait quarante centimètres de haut. » Ce jour-là, Chéreau a expliqué aux comédiens qu'il leur arrivait de se tromper dans le texte, que les techniciens eux aussi avaient le droit à l'erreur. Le travail ne se limitait pas à exécuter des consignes, l'objectif esthétique était formulé, compris et interprété. "On ne faisait pas ça bêtement, explique Bernard Steffenino, qui était aussi constructeur, Chéreau nous disait : "Avec cet effet-là, je veux arriver à ça." Il suffisait de le savoir, on pouvait imaginer un système différent : modifier la machinerie, "trouver un truc". » Une pensée en acte qui rappelle le « sens du dramatique » prêté aux machinistes par Louis Jouvet.

\section{Évolutions de la régie}

Nous avons choisi d'évoquer pour terminer ce que nous avons appelé les « minirégies ». Le terme désigne ici un poste de commande (du son ou de l'éclairage), de taille plus modeste qu'une régie habituelle et implanté à un endroit original du lieu théâtral.

a) Un régisseur dans les coulisses

La régie lumière du spectacle était classiquement installée dans la salle, différemment selon les théâtres (fig. 5), comme l'étaient aussi les deux poursuiteurs. Restait à éclairer à l'aide de fluos placés au sol le cyclo de plastique disposé à l'arrière de la forêt, de façon

20. Formule de Jean-Pierre Léonardini, texte accompagnant les photos de Claude Bricage pour un projet d'ouvrage non réalisé, 1977 [p. 5], IMEC/Fonds P. Chéreau. La Dispute.

21. Pour cette citation et les suivantes, B. Steffenino à M.-M. Mervant-Roux, entretien cité. 
à recréer l'alternance des jours et des nuits. Un petit échafaudage avait été construit au lointain, côté jardin, en lisière de la forêt, sur lequel était installée une régie spécialisée. Gérard Boucher y grimpait pour chaque effet d'éclairage. En plus de la manipulation des lampes fluo, il gérait la poursuite qui accompagnait les acteurs à l'intérieur de la forêt.

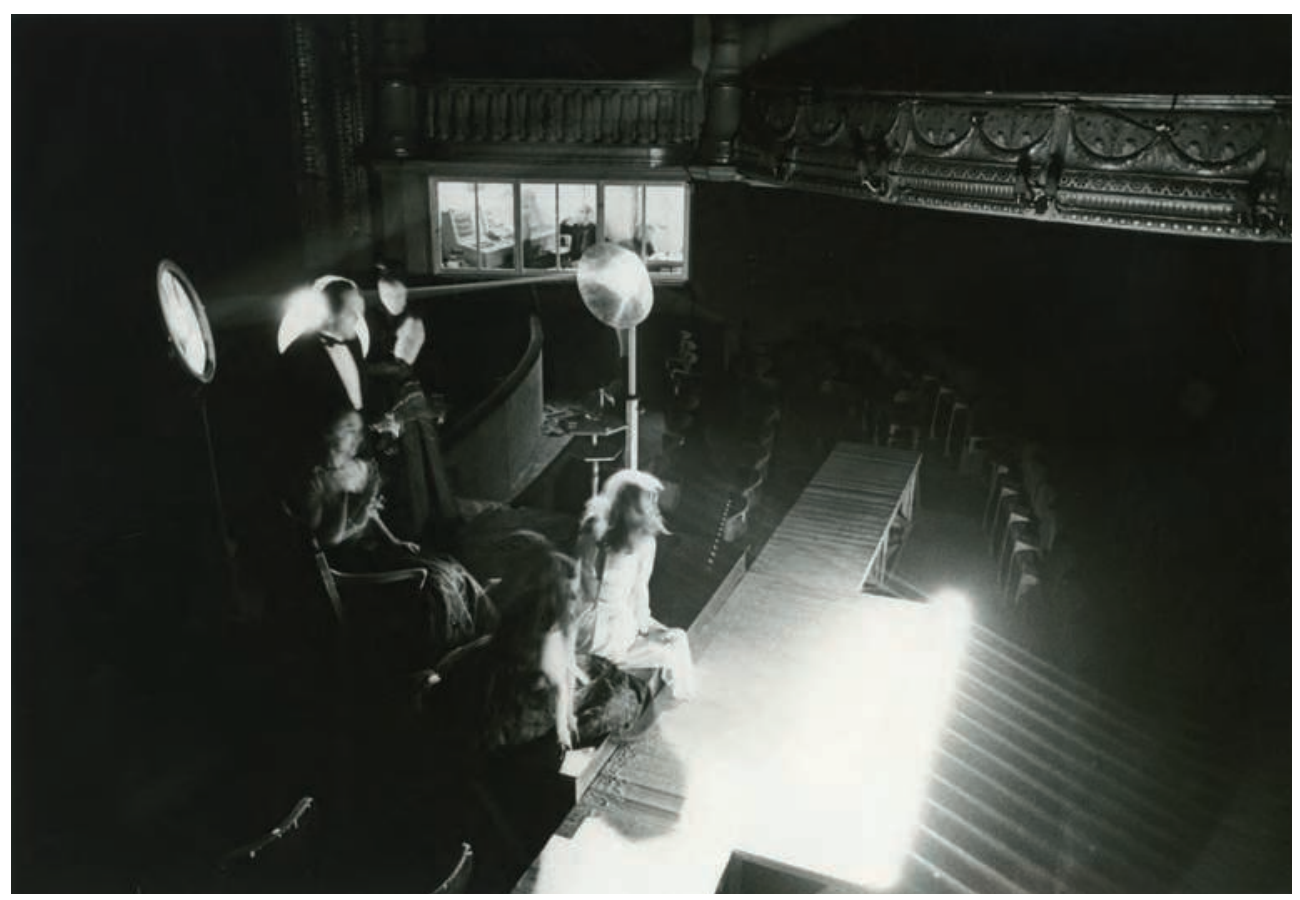

Fig. 5 : La Dispute. Le praticable du prologue, avec de part et d'autre le couloir d'accès et la régie lumière, installée dans une loge de côté. Théâtre de la Musique, 1973

Photographie de Nicolas Treatt (C) Nicolas Treatt

b) Un régisseur dans le décor

Après s'être tenu avec ses trois magnétophones dans la cabine de régie, André Serré avait trouvé le moyen de s'installer sur le plateau, extrêmement près des comédiens dont il accompagnait en permanence les mouvements et les voix par des cris d'animaux ou des séquences de musique. Il «s'est inventé » (sic) un petit couple de micros, et il écoutait le spectacle au casque : ses oreilles se trouvaient ainsi « au niveau du premier balcon », luimême était assis dans la première «maison » côté jardin (cette maison ne bougeait pas). Invisible, il ne voyait presque rien de la scène, ne disposant que d'une petite fenêtre, mais il entendait tout et il était au cœur du jeu.

« [L]'invention, écrit Gilbert Simondon, se distingue des images qui la précèdent par le fait qu'elle [...] ne reste pas dans l'être vivant, comme une part de l'équipement mental, mais enjambe les limites spatio-temporelles du vivant pour se raccorder au milieu qu'elle organise 22 . »

22. Gilbert Simondon, Imagination et invention. 1965-1966, Paris, PUF, 2014, p. 185-186. 
Le passage - extrêmement discret - des deux techniciens-régisseurs dans des secteurs a priori interdits du plateau, quasiment au contact du décor et des comédiens, nous semble traduire dans l'espace la proximité généralisée entre "l'inventivité " technique et la «création » artistique (fig. 6). C'est maintenant à la reconnaissance de la régie comme pôle autonome de la genèse théâtrale que nous allons consacrer la suite de notre réflexion.

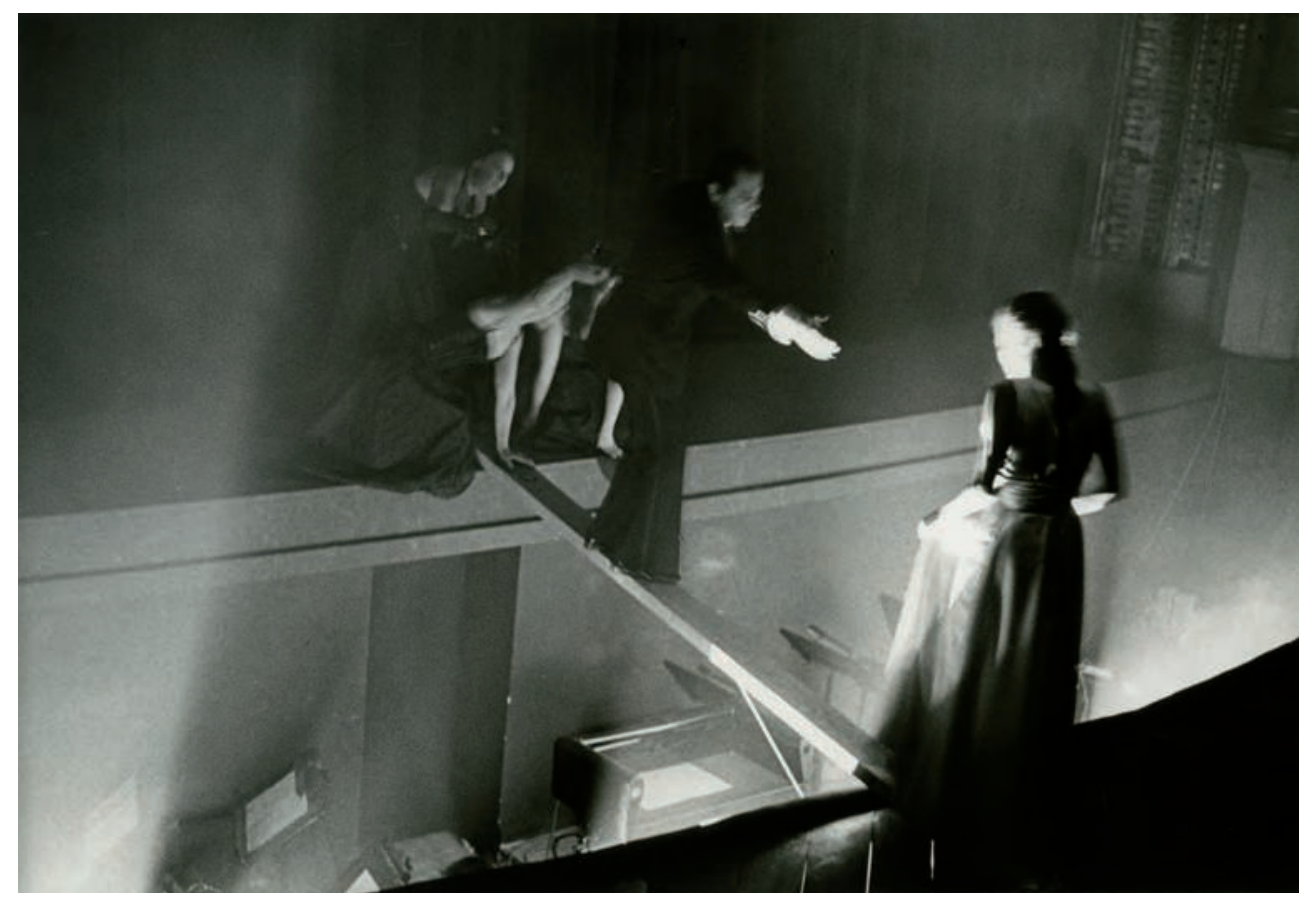

Fig. 6 : La Dispute. La planche au-dessus de la fosse d'orchestre. Comédiens et techniciens coopèrent pour l'installer et la faire disparaître. Théâtre de la Musique, 1973

Photographie de Nicolas Treatt @C Nicolas Treatt

\section{L'autorité de la technique}

Dans un entretien effectué le 15 janvier 1973 pour la revue militante Travail théâtral, Émile Copfermann évoque avec Patrice Chéreau le théâtre populaire « où tout le monde, dit-il, devrait avoir pouvoir sur la création ». « On ne peut pas donner de forme démocratique à un travail théâtral, à une répétition », répond le metteur en scène. «La seule collaboration qu'on puisse avoir avec un technicien est qu'il accomplisse ce qu'on lui demande 23 . » Le modèle auquel se réfère Copfermann est celui de la création collective, dont l'un des principes consiste à conférer aux praticiens de la régie la fonction créative, ce qui signifie déposséder le metteur en scène de cette fonction et la distribuer «démocratiquement » dans le groupe. Chéreau semble au contraire défendre

23. «La mousse, l'écume », Travail théâtral, n XI, avril-juin 1973, p. 3-26 ; p. 24. 
l'idée d'une hiérarchie naturelle entre le créateur (le metteur en scène) et le technicien, assigné à obéir et à accomplir la tâche imposée. Nous avons pu vérifier sur l'exemple de La Dispute que loin d'être de dociles exécutants, les techniciens qui à cette période travaillent pour ses spectacles développent des activités extrêmement inventives, parfois comparables à celle des comédiens, du point de vue de la performance physique, de la sensibilité et de l'imaginaire en jeu. Le metteur en scène, cependant, qui apparaît comme la figure unificatrice de l'ensemble, semble exercer un pouvoir absolu sur sa double « distribution ». Nombreux sont alors les portraits de Chéreau en chef de bande rimbaldien, nombreux les témoignages d'acteurs sur sa séduction physique et humaine. Cette séduction s'exerçait aussi sur les techniciens : l'effet de son regard - «Patrice rentrait en vous » (André Serré) -, l'effet de sa voix ${ }^{24}$, de son autorité charismatique de «meneur d'hommes » (Bernard Steffenino) sont souvent évoqués. Certes, il rend les gens talentueux, mais au prix d'une soumission à sa seule autorité ? Les choses ne sont pas si simples.

\section{Le domaine réservé de la technicité}

Une des leçons les plus précieuses des entretiens est que la compétence de Chéreau pour évaluer le travail fait et le travail possible était indéniable - «Il sait très bien comment fonctionne un cintre, même s'il ne sait pas le faire, il sait jusqu'où il peut aller, il a la notion de l'ouvrage »(B. Steffenino) -, que son savoir était suffisant, mais pas plus : le metteur en scène n'empiétait pas sur le domaine des techniciens. Même dans le champ de l'éclairage, où il était capable de remplacer le régisseur au pied levé (cela lui est arrivé pendant la tournée de La Dispute à Belgrade, Gérard Boucher étant tombé malade), il entretenait avec le spécialiste un rapport respectueux - ce qui transparaissait dans la suite de sa réponse à Émile Copfermann : "Si ce qu'on lui demande [au technicien] ne marche pas, qu'il propose autre chose. » Traduisons : si le metteur en scène ne maîtrise pas assez la technique pour concevoir convenablement un élément du spectacle, c'est au technicien d'inventer une solution, et éventuellement de modifier l'élément prévu. «Il s'abreuvait de nos idées », résume Gérard Boucher. Et la confiance était totale. Une fois que le technicien avait évalué le temps qu'il lui fallait pour résoudre un problème, Chéreau ne s'en occupait plus. " Il ne vous rappelait pas toutes les deux minutes. Jamais il ne disait : "Tu en es où ?" "(B. Steffenino). Même si le metteur en scène semblait décider de tout, chacun reconnaissait dans l'œuvre ce qui était venu de lui, et cette part lui était reconnue. Moins à travers l'amélioration des conditions de travail (des salaires et horaires convenables, pour une charge de travail très lourde, et acceptée) que par des gestes symboliques. Celui d'exiger, par exemple - on en déduit que c'était rare - que les techniciens fussent invités aux réceptions organisées lors des tournées, au même titre que les comédiens.

24. «Patrice me donnait le texte, mais il commençait par me le raconter. Et il raconte bien, Chéreau ! Vraiment bien », Y. Bernard, loc. cit., p. 8. 


\section{La force des "gens de métier »}

Les techniciens du Piccolo Teatro de Milan sont des gens prodigieux. Ça vient de ce que l'Italie est un pays plus pauvre, où le sens du métier existe encore 25 .

Ce qui se dégage aussi des témoignages est que la plupart des praticiens de La Dispute étaient adossés à des cultures professionnelles non théâtrales, ou autrement théâtrales : celle du bâtiment pour beaucoup, celle du cinéma (ou de la télévision), celle de la machinerie à l'italienne ${ }^{26}$. Bernard Steffenino, par exemple, était serrurier de métier avant son embauche au Théâtre de Villeurbanne en 1972. C'est même parce qu'il était le seul vrai serrurier du théâtre et qu'il y avait un décor en construction pour Roger Planchon qu'il n'a pas accompagné La Dispute à Paris à sa création. Il raconte comment les trois nouveaux directeurs du TNP, Planchon, Chéreau et, selon lui, surtout Gilbert, ont su «monter une structure nouvelle, avec un super directeur technique et un chef constructeur extraordinaire », comment ils ont cherché de «vrais professionnels », « pas de la bidouille de théâtre », comment pendant quelques années, le théâtre a ainsi produit « les plus beaux spectacles de France ». Bernard Steffenino avait ses critères propres pour évaluer les réalisations auxquelles il participait. Son avis sur le savoir-faire des Italiens était nuancé. La légèreté, dit-il, avait son revers. De telles compétences donnaient aux techniciens une grande fierté et une certaine liberté par rapport au monde du théâtre. La culture syndicale, parfois militante, d'une partie des personnels de Sartrouville et de Villeurbanne a sans doute aussi contribué à la force de la régie, mais un grand nombre de pratiques demeuraient strictement solitaires. «Il n'y a pas création collective, et le travail en est d'autant plus collectif 27 . » Cette formule de Chéreau à propos de l'équipe artistique vaut aussi pour l'équipe technique, et décrit même très bien son mode créatif. Le dernier facteur est générationnel : ceux dont nous parlons ont pour la plupart entre 20 et 30 ans. Ils sont euxmêmes recréés par cette aventure, comme leurs parcours ultérieurs en témoignent.

Ainsi, tout se passe comme si, en face de l'autorité esthétique, se manifestait une autre autorité, d'un autre ordre, comme si Patrice Chéreau, pour des raisons en partie liées à son itinéraire personnel, en partie à la vitalité de certaines formes de culture politique dans la vie théâtrale du temps, reconnaissait, après Jouvet, Vilar et quelques autres, l'importance de la technique et sa place structurelle dans la création théâtrale.

Cette position non académique assumée par des hommes de théâtre majeurs n'a pas suscité d'importants travaux théoriques. Nous relèverons avec d'autant plus d'intérêt ce qu'écrit Marcel Freydefont dans l'article «Régie »du Dictionnaire encyclopédique du théâtre à travers le monde. Après avoir rappelé que le mot « régisseur » a pu désigner au XIX ${ }^{\mathrm{e}}$ siècle celui qui - il cite Pougin - «monte les ouvrages, [...] en règle la marche scénique, donne aux acteurs les indications », Freydefont souligne que « la régie finit par s'imposer comme un domaine à part entière qui pose cependant de multiples problèmes :

25. P. Chéreau à E. Copfermann, « La mousse, l'écume », loc. cit.

26. «J'avais cherché des gens qui n'étaient pas forcément des gens de théâtre. Ils appartenaient à beaucoup de corps de métier, et même du bâtiment. C'étaient de très bons artisans », Y. Bernard, loc. cit., p. 6.

27. «La mousse, l'écume », loc. cit. 
théoriques, car si aujourd'hui la dramaturgie et la scénographie définissent des domaines reconnus [...], la régie doit leur être adjointe tout aussi fondamentalement comme constitutive du théâtre (nous soulignons) ; esthétiques, car la régie peut répondre à deux types de situation : soit une position de subordination et d'exécution technique, soit une position de conception et de gestion pratique [...] dont on peut considérer historiquement la mise en scène comme un cas de figure (nous soulignons) 28 ».

Le plaidoyer de Gilbert Simondon en faveur d'une véritable reconnaissance intellectuelle de la technique semble trouver au théâtre un champ d'application privilégié, son analyse de la technicité venant éclairer ce qui constituait notre interrogation seconde, concernant le caractère collectif supposé de "l'équipe » des techniciens. Selon ce philosophe, en effet, la technique « est du collectif», mais ce qu'il veut dire par là ne correspond pas aux notions de « création collective » ou de « collectif de création » :

[A]u-dessus de la communauté sociale de travail, au-delà de la relation interindividuelle qui n'est pas supportée par une activité opératoire, s'institue un univers mental et pratique de la technicité, dans lequel les êtres humains communiquent à travers ce qu'ils inventent. L'objet technique [...] devient le support et le symbole de cette relation que nous voudrions nommer transindividuelle 29 .

Dans les propos des techniciens de La Dispute, une image plusieurs fois apparue, celle de l'atelier d'artisans, traduit, nous semble-t-il, quelque chose de cette idée selon laquelle le collectif naîtrait de l'invention elle-même. L'espace imaginaire de l'atelier, emprunté au passé mythique de plusieurs arts nobles et voisins : la peinture, l'architecture, la décoration d'opéra ${ }^{30}$, a constitué le cadre transitionnel et transindividuel heureux de la genèse de La Dispute.

Nous proposons l'introduction dans les genèses théâtrales, au côté du pôle dramatique, incarné par l'auteur, et du pôle scénique, incarné par le metteur en scène, d'un troisième pôle, le pôle technique - souvent, personne ne l'incarne, l'auctorial reste collectif. Un tel ajout exigerait une refondation méthodologique. Celui-ci se caractérise en effet par la pluralité et la diversité des praticiens, l'importance des conventions tacites, la rareté des traces écrites directes du travail, puisqu'on y use beaucoup de l'oral, et surtout on fait et on montre. D'où la nécessité d'en constituer le dossier génétique à partir de traces indirectes, de souvenirs des participants - ou de l'enquête ethnographique pour les spectacles contemporains.

28. M. Freydefont, « Régie », Dictionnaire encyclopédique du théâtre à travers le monde, dir. Michel Corvin, Paris, Bordas, 2008.

29. Gilbert Simondon, Du mode d'existence des objets techniques, Paris, Aubier, 2012, p. 335.

30. Voir Rémy Campos, Aurélien Poidevin, La Scène lyrique autour de 1900, Paris, L'Eil d'or, 2011. 
Marie-Madeleine Mervant-Roux est directeur de recherche émérite au CNRS (études théâtrales). Ses travaux sur le spectateur et sur les théâtres d'amateurs s'inscrivent dans une recherche sur la fonction dramatique en Europe. Elle a coordonné avec Jean-Marc Larrue (Université de Montréal) un programme sur « Le son du théâtre (XIX $\mathrm{X}^{\mathrm{e}} \mathrm{XXI}{ }^{\mathrm{e}} \mathrm{s}$.) » et dirige le projet ANR ECHO [ÉCrire l'Histoire de l'Oral] «L'émergence d'une oralité et d'une auralité modernes. Mouvements du phonique dans l'image scénique (1950-2000) ».

marie-madeleine.mervant-roux@cnrs.fr

\section{Résumés \\ Construire à plusieurs la machine à jouer. Figures de l'invention technique dans La Dispute mise en scène par Patrice Chéreau}

Le théâtre est une pratique collective. Ses différentes fonctions (définition d'un espace à part, élaboration d'un matériau textuel, élaboration d'un jeu scénique) sont rarement assumées par une seule personne. La critique génétique, après avoir intégré à côté de l'auteur de l'œuvre dramatique la figure du metteur en scène et pris acte de l'hétérogénéité de toute production, n'a toutefois pas pris en compte l'organisation matérielle de la représentation, la « régie », négligée par les études théâtrales elles-mêmes. Les constructeurs, machinistes, électriciens, techniciens et régisseurs sont appréhendés de façon floue comme un ensemble d'exécutants occupés à une activité foncièrement coopérative. Faisant l'hypothèse que leur fonction n'est pas de simple exécution, nous prendrons aussi de la distance par rapport à une deuxième idée reçue : le caractère « collectif » de leur intervention. Notre exemple sera La Dispute (mise en scène de Patrice Chéreau, 1973). Avec une question de méthode : comment établir ce volet du dossier génétique en l'absence de traces écrites?

Theater is a collective practice. Its different functions (the definition of a separate space, the elaboration of a textual material and staging) are rarely assumed by only one person. Genetic criticism, after having integrated, beside the play's author, the figure of the stage director and taken into account the heterogeneity of any production, has nonetheless not yet examined the performance's material organization, the stage management, neglected even by theater studies themselves. Builders, stagehands, electricians, technicians and stage managers are vaguely perceived as a group of subordinates involved in an essentially cooperative activity. Working on the hypothesis that their function is not a mere execution, we will also distance ourselves from the second preconception, the "collective" character of their intervention. We will take as our example La Dispute (directed by Patrice Chéreau, 1973). With the question of method: how can we establish this stage of the genetic dossier in the absence of written traces?

Das Theater ist eine kollektive Praxis. Seine unterschiedlichen Funktionen (Festlegung eines eigenen Raumes, Erarbeitung des Textmaterials, Erarbeitung der szenischen Darbietung) sind zumeist nicht in ein und derselben Person vereinigt. Die genetische Kritik hat neben der Rolle des Autors eines Dramas die Rolle des Regisseurs zu würdigen verstanden und insofern die Heterogenität jeder (dramatischen) Produktion erkannt, jedoch wurde bislang die materielle Basis einer Aufführung, einer Regie, vergleichsweise vernachlässigt. Die Bühnenbildner, Maschinisten, Elektriker, Techniker und Regisseure werden in unbestimmter Weise als ein Ensemble von Ausführenden angesehen, die in einer zutiefst kooperativen Tätigkeit begriffen sind. Indem wir von der Hypothese ausgehen, dass ihre Funktion eben nicht nur einfach im „Ausführen“ besteht, distanzieren wir uns zugleich von einer zweiten gängigen Vorstellung: nämlich vom „kollektiven“ Charakter ihres Einsatzes. Das Beispiel, das wir für unsere Untersuchung heranziehen, ist La Dispute (im Jahre 1973 inszeniert von Patrice Chéreau). Um es methodologisch auszudrücken: wie kann man jenen Teil der genetischen Untersuchung auf eine verlässliche Basis stellen, wo geschriebene Spuren fehlen?
I 1 teatro è una pratica collettiva. Le sue differenti funzioni (definizione di uno spazio, elaborazione di un testo, elaborazione di un'interpretazione scenica) sono raramente espletate da una sola persona. Se la critica genetica ha integrato la figura del regista accanto a quella dell'autore dell'opera drammatica, prendendo atto dell'eterogeneità di ogni produzione, non ha tuttavia ancora preso in considerazione l'organizzazione materiale della rappresentazione, trascurata anche dagli stessi studi teatrali. I costruttori, macchinisti, elettricisti, tecnici e direttori di scena vengono approssimativamente considerati come un insieme di esecutori che si occupano di un'attività sostanzialmente cooperativa. Con l'ipotesi che la loro funzione non sia semplicemente esecutiva, prenderemo anche le distanze rispetto a un altro luogo comune: il carattere "collettivo" del loro intervento. Il nostro esempio sarà La Dispute (messa in scena di Patrice Chéreau, 1973). Con una domanda riguardo al metodo: come stabilire questa parte del dossier genetico in mancanza di tracce scritte?

O teatro é uma prática coletiva. As suas diferentes funções (definição de um espaço próprio, elaboração de material textual e de jogo cénico) raramente são executadas por uma pessoa só. A crítica genética emparelha com o dramaturgo a figura do encenador e reconhece a heterogeneidade que caracteriza qualquer produção. Contudo, não tem em consideração os aspectos da organização material da representação, a "régie", que é negligenciada pelos próprios estudos de teatro. Construtores, maquinistas, eletricistas, técnicos e registas são vagamente percebidos como um conjunto de artistas empenhados em atividades basicamente de colaboração. Ao defender a hipótese de que sua função não é meramente de execução, também nos afastamos de um segundo erro comum: o carácter «colectivo» da sua intervenção. Damos como exemplo La Dispute (encenada por Patrice Chéreau em 1973). Com um problema de método : como estabelecer esta secção do dossier genético na ausência de documentos escritos?

El teatro es una práctica colectiva. Sus diferentes funciones (definición de un espacio particular, elaboración de un material textual, elaboración de un juego escénico) rara vez son asumidas por una misma persona. La crítica genética, después de haber integrado, junto con el autor de la obra dramática, la figura del director y tomado en cuenta la heterogeneidad de toda realización, no se ha detenido a considerar, sin embargo, la organización material de la representación, la "producción", desestimada aún por los propios estudios teatrales. Los constructores, maquinistas, electricistas, técnicos y directores de escena son percibidos de manera difusa como un conjunto de ejecutantes dedicados a una actividad esencialmente cooperativa. Formularemos aquí la hipótesis de que su función no es simplemente de ejecución y al mismo tiempo tomaremos distancia con respecto a una segunda idea preconcebida: el carácter "colectivo" de su intervención. Nuestro ejemplo será La Disputa (puesta en escena de Patrice Chéreau, 1973) y nuestro interrogante metodológico será: ¿cómo establecer este elemento del dossier genético sin contar con rastros escritos? 